\title{
A review of studies on the COVID-19 epidemic crisis with a preventive approach
}

\author{
Maryam Feiz Arefi ${ }^{\mathrm{a}, \mathrm{b}}$ and Mohsen Poursadeqiyan ${ }^{\mathrm{a}, \mathrm{b}, *}$ \\ ${ }^{a}$ Department of Occupational Health Engineering, Torbat Heydariyeh University of Medical Sciences, \\ Torbat Heydariyeh, Iran \\ ${ }^{\mathrm{b}}$ Health Sciences Research Center, Torbat Heydariyeh University of Medical Sciences, Torbat Heydariyeh, Iran
}

Received 10 June 2020

Accepted 21 June 2020

\begin{abstract}
.
BACKGROUND: COVID-19 is a highly contagious acute respiratory syndrome and has been declared a pandemic in more than 209 countries worldwide. At the time of writing, no preventive vaccine has been developed and tested in the community. This study was conducted to review studies aimed at preventing the spread of the coronavirus worldwide.

METHODS: This study was a review of the evidence-based literature and was conducted by searching databases, including Google Scholar, PubMed, and ScienceDirect, until April 2020. The search was performed based on keywords including "coronavirus", "COVID-19", and "prevention". The list of references in the final studies has also been re-reviewed to find articles that might not have been obtained through the search. The guidelines published by trustworthy organizations such as the World Health Organization and Center for Disease Control have been used in this study.

CONCLUSION: So far, no vaccine or definitive treatment for COVID-19 has been invented, and the disease has become a pandemic. Therefore, observation of hand hygiene, disinfection of high-touch surfaces, observation of social distance, and lack of presence in public places are recommended as preventive measures. Moreover, to control the situation and to reduce the incidence of the virus, some of the measures taken by the decision-making bodies and the guidelines of the deterrent institutions to strengthen telecommuting of employees and reduce the presence of people in the community and prevent unnecessary activities, are very important.
\end{abstract}

Keyword: Pandemic, prevention, novel coronaviruses, COVID-19

\section{Introduction}

Coronaviruses are a large family of viruses and a subcategory of Coronaviridae, which includes different viruses from the common cold to more serious diseases such as SARS, MERS, and COVID-19. The virus naturally spreads in mammals and birds.

${ }^{*}$ Corresponding author: Mohsen Poursadeqiyan. E-mail: mo. poursadeghiyan@uswr.ac.ir.
However, seven human-transmitted coronaviruses have been discovered so far [1]. Coronaviruses are zoonotic; that is, they are transmitted between animals and humans [2]. The latest species, the new corona, became widespread in Wuhan, China, in December 2019, and studies suggest that this disease has an animal origin, which was first transmitted from animal to human, and then become a pandemic through human-to-human transmission in many countries [3]. Given the large number of 
people who have been in contact with seafood and have been to the wet animal wholesale market in Wuhan, it is possible that this market is a zoonotic zone of COVID-19. Numerous studies have been conducted to identify the root cause of the disease, but so far the cause has not been precisely determined, and the results only show that mammals are most likely the link between COVID-19 and humans [1]. Based on the results of the virus genomes sequencing and evolutionary analysis, bats have been suspected as the natural host of the virus [4], and COVID-19 may have been transmitted to humans through unknown intermediate host sources for infection. According to the protein sequences alignment and phylogenetic analysis, it is more likely to provide an alternative intermediate host, such as turtles, snakes and pangolin [5]. It seems that discovering the origin and how it was created will help scientists to prohibit the use of the category of wild animal meat for preventing the occurrence of these dangerous global phenomena. It seems that this method is the most basic way to stop the disease and prevent such dangerous epidemics, although there have been some hypotheses that the virus may be man-made, of which the results of the study conducted by Menachery et al. have made this hypothesis more serious [6]. However, in a recent study by Andersen published in Nature, the hypothesis that COVID-19 was man-made has been rejected [7]. According to statistics provided by China, the death rate caused by COVID-19 has been higher in people over the age of 50 [8]. In a small number of patients, the virus quickly led to developing acute respiratory symptoms and organ failure, and even death. Some adults and children with COVID-19 may not have severe symptoms, but they can transmit the virus to other adults [9]. Studies by Qifang et al. have shown that the risk of being infected by COVID-19 in children and adults is equal, although fewer children have severe symptoms [10].

At the time this study was conducted (in early April 2020), more than one million people worldwide have been infected by the disease, and the number will certainly increase [11]. There have been reports that younger people were also among the victims of COVID-19. For instance, a 24-year-old Iranian nurse, three teenagers aged 12,14, and 16 in Europe, and a 16-year-old girl in France died due to the virus without any medical history $[12,13]$. There have been reports of a positive test for COVID-19 in some pets, including two dogs of different breeds, a cat from Hong Kong, and a Malaysian tiger infected at a zoo in the USA $[14,15]$. Studies have shown that the period from symptoms onset of COVID-19 to death ranged from 6 to 41 days, with an average of 14 days. This period depends on the person's age and immune system, and this period is lower among adults over 70 than in those under 70 [1]. The results of the study by Kampf et al. showed that $30 \%$ of the carriers were asymptomatic, only $43.8 \%$ of the individuals had a fever, and in $15.7 \%$ of the cases severe pneumonia was observed [8]. On the other hand, the results of epidemiologic studies have shown that every person with COVID-19 can infect a large number of people. Thus, if the process of stopping the chain of infection by the application of preventive measures in the community is ignored, COVID-19 will always have a host, and the number of infected people will exponentially increase. By April 2020, more than 200 countries (i.e. almost the entire world) have been affected by COVID-19 [11]. Statistics show that human casualties in China have been lower than in many other countries, which is one of the reasons for the success in the prevention of COVID-19 [16]. The virus is transmitted through contact with respiratory droplets caused by coughing or sneezing or by touching objects and surfaces that contain droplets of coughs or sneezes of an infected person. Studies have also shown that coronaviruses can survive on environmental surfaces and inanimate objects for several days [17]. Due to the fact that no preventive vaccines have been developed and tested in the community at the time of writing, the present study intended to review studies with the aim of preventing the COVID-19 crisis worldwide.

\section{Materials and methods}

This study was based on an evidence-based literature review and was conducted by searching databases, including Google Scholar, PubMed, and ScienceDirect, by April 2020. The search was based on keywords, such as "Coronavirus", "COVID-19", and "prevention". The reference list of the final study was also reviewed to find articles that had not been obtained through the search. The guidelines published by reputable organizations such as the World Health Organization (WHO) and the Center for Disease Control (CDC) have also been used in this study. Studies published in English and conducted in different countries were selected for analysis. Studies and research related to treatment and clinical diagnostic symptoms have been excluded from the study. Finally, after removing duplicate cases, the search 
results were independently reviewed by two authors based on the inclusion and exclusion criteria, and the studies that entered the final analysis phase were extracted and analyzed.

\section{Discussion and results}

Based on the results obtained from different articles, the results are categorized in several categories with the prevention approach.

\subsection{Disease screening in the workplace}

Disease screening is a critical and important step for detecting and isolating infected people. In this plan, governments and their health centers should take real and accurate tests from possibly infected people and intensify the screening rate as much as possible. By implementing this plan, patients are recognized more quickly and are separated from the work- and family environment, and are treated and quarantined entirely [18]. In this case, when the COVID-19 test is positive, the family of the infected person, as well as people and colleagues who have been in contact with this person during the last two weeks are also quarantined to prevent the transmission of the infection to other people in the community. Without a doubt, this is the most important way to stop the global epidemic chain [19]. However, the monitoring should continue until the end of the epidemic and eradication of the disease, and no person will be safe, even when the test is negative, and it is possible that the test, through exposure to the virus in the next days, with the appearance of suspicious symptoms, becomes positive. This strategy, known as eliminating the risk, is the most effective and efficient risk reduction strategy [20]. Some of the challenges for the screening of diseases include the shortage of diagnostic kits and the duration of the test, the amount of use and the time required for taking the test, as well as the cost to governments. Some countries, such as Iran, monitor the health condition of people by establishing an online self-reporting site and telephone inquiry system (4030 system). Although this is one of the least effective ways to diagnose patients and has been somewhat successful in performing this task, the incidence of diagnostic symptoms is extremely challenging. The incubation period of at least 14 days and the probability of being asymptomatic carriers are among the many challenges of this part-time diagnostic method. On the other hand, people have been conditioned to observe the distance from people with symptoms, and all these cases have played a role in the hidden spread of COVID-19.

The source of the COVID-19 outbreak may be likened to a mountain of ice; the part that comes out of the water represents people with the symptoms, and the part underwater, that is probably much larger, represents the asymptomatic carriers. Therefore, it is always recommended to pertain social distance and cover the respiratory areas as a precautionary measure. In some work environments such as production halls and assembly lines, a large number of employees may be present in a closed environment, so screening and disease detection in such environments have particular importance because it will prevent the transmission of the disease to colleagues.

\subsection{COVID-19 transmission and pathogenicity}

The incubation period for COVID-19 is 1-14 days, and during this time, the person can transmit the disease to others [5]. It should be noted that the presence of a symptom does not indicate the presence of the disease and is not the condition for caution, but the observance of principles of health and prevention should always be taken into consideration. Results of studies conducted by Bai et al. showed that $30 \%$ of the carriers are asymptomatic and can carry the virus for up to two weeks. 55\% of the patients have mild symptoms that began less than a week after infection and improved after a few days. These patients are carriers of the virus for up to three weeks. About $10 \%$ of the patients show severe symptoms, and their symptoms began late in the first week; this group carries the disease for about four weeks. About $5 \%$ of the patients show very severe symptoms, which occur after a few days; their breathing becomes very difficult, they need to be transferred to the ICU and need a ventilator, and, eventually, 50\% of this group recovers [21]. Only some infected people had symptoms such as a runny nose, a cough or sneeze, sputum, etc. [22]. $43 \%$ of the people had fever symptoms [8].

COVID-19 has the ability to stay on different surfaces from two hours up to nine days, depending on the type of surface, temperature, and relative humidity [23]. The results showed that the virus persistence was higher on steel and glossy surfaces [8]. COVID19 is transmitted through contact with droplets of the infected person's respiratory tract, which may come in direct contact with the person's face or are 
precipitated on surfaces, and lead to infection of other people through contact with infected surfaces. Some studies in China have shown that the virus is also found in the feces of an infected person, which indicates that it is transmitted in different ways [5]. Airborne transmission of COVID-19 has also been discussed. The WHO reported that the virus could be suspended for up to eight hours [24]. COVID-19 is still alive three hours after being spread through tiny droplets, according to a recent study by the American Institute for Allergy and Infectious Diseases Research. In simpler terms, very small particles that come out of the mouth and nose when sneezing or coughing carry the virus and can be the disease carrier for three hours [25]. According to research published in the Journal of Virology, people should never shake used or unused clothes, plates, or fabrics because the virus is highly ineffective when attached to a porous surface, and only lasts three hours on porous fabrics and materials, four hours on copper (this is a natural disinfectant substance) and wood, 24 hours on cardboard, 42 hours on metal and 72 hours on plastic.

However, if the materials are shaken, the virus molecules float in the air for up to threehours and can settle in the nose [26]. Examination of the room of three COVID-19 patients in Singapore showed that there was no virus in the air of the patient rooms, which can indicate that the virus was not airborne. However, more samples are needed. Even in presence of severe infection, routine cleaning and disinfection of patients' rooms are sufficient to eradicate the virus [27]. Due to the airborne capability of COVID-19, it is recommended to reduce the activites and processes that intensify the production of aerosol particles in the workplace, including the use of compressed air to clean surfaces, and, if necessary, this process should be done in isolation and with the use of appropriate masks in order to reduce the exposure to production aerosols.

In the section, the role of masks in preventing the transmission of the virus from the patient is discussed. Reactions such as coughing and sneezing by an infected person or carrier can cause indoor air pollution or pollution of the surfaces [17]. Therefore, the use of masks can effectively prevent the spread of COVID-19, and some countries have emphasized the use of masks for healthy people and patients alike [28]. Some sources have suggested that, in the absence of a mask, people should use a handkerchief in front of the mouth, the elbow or the hand to cover the mouth when coughing or sneezing $[29,30]$.

\subsection{Carriers and hosts}

Some studies have suggested that wild animals were the primary origin of COVID-19, as its primary reservoir and intermediate host were identified to be a bat and anteater, respectively [3, 4]. But so far, the transmission of COVID-19 from animals to humans has not been shown. A few cases of domestic animals such as dogs and cats were recently added to the list of carriers $[14,15]$, which can be very worrying during a global pandemic. WHO's initial recommendations suggested to avoid animals and not get in contact with animals [31]. Based on these interpretations, the person-to-person transmission of the disease is the most important manner for its transmission, which can be prevented by observing a safe distance of 2 meters. Studies have shown that it is also possible to transmit the disease from asymptomatic carriers, and people are the most dangerous carriers [32]. In fact, the most important carriers are humans who have made it impossible to break the disease chain because of their contact and close social relationships in public places. Due to the transmissibility of the disease from animals to humans and, recently, humans to animals, occupations involving animals such as sellers of live animals, veterinarians, zoo staff, ranchers, farmers and slaughterhouses and all people who may have contact with domestic or wild animals should avoid any direct or unprotected contact.

\subsection{Observance of the personal hygiene principles}

It is important to was the hands properly and avoid contact with surfaces. Due to the structure of COVID19 , which has a lipid membrane, the use of soap and water is effective in destroying the membrane and the the virus. Frequent handwashing with soap during the day, especially after contact with high-touch surfaces, after defecation and before eating, as well as after sneezing, coughing and blowing the nose are recommended. If soap is not immediately available, 60-90\% alcohol-based detergents are suggested [33]. Some sources recommend that people should continue washing their hands for 20 seconds or more. By dissolving the lipid layer, the protein molecule is decomposed on its own. The heat melts the lipid, so hands should be washed with water with a temperature above $25^{\circ} \mathrm{C}$ and clothes should be washed above this temperature as well. In addition, hot water creates more foam, and therefore heat is more useful [26]. The time recommended by WHO in clinical settings 
for alcohol-based hand rub [ABHR] is $20-30$ seconds, and the volume is $1.5-2.25 \mathrm{ml}$ [34]. According to $\mathrm{WHO}$, the use of gloves is recommended in public places and clinical centers for the care of COVID-19 patients. All members of the clinical staff, laboratory technicians, cleaning staff, and visitors are advised to wear disposable gloves. For general use in clinical settings, gloves should be made of nitrile or latex, should be packed, sterile, and disposable without pores [2], and should be discarded after use [23].

In a study published in The Lancet Gastroenterology and Hepatology, out of 74 patients with positive respiratory swabs, stool samples were positive for 41 cases. In these patients, the respiratory sample was positive for an average of 16.7 days, but the stool sample was positive for an average of 27.9 days. This means that the stool sample was positive for an average of 11.2 days longer than the respiratory sample. At present, the decision to discharge the patient is based on the absence of symptoms and the presence of two consecutive PCR tests of sputum or airway at a time interval of more than 24 hours. However, this study shows that the virus is actively reproducing in the gastrointestinal tract, and oral-fecal transmission is possible even after a negative respiratory sample [35]. Studies have also shown that coronaviruses can survive on peripheral surfaces and inanimate objects for several days [17], so the disinfection of high-touch surfaces is essential. According to the results of previous studies, disinfectants such as $62-71 \%$ ethanol, $0.5 \%$ hydrogen peroxide, or $0.1 \%$ sodium hypochlorite can effectively inactivate coronaviruses within one minute, but other disinfectants such as $0.05-0.2 \%$ benzalkonium chloride or $0.02 \%$ chlorhexidine digluconate were found to be less effective [8]. Alcohol is recommended only for the disinfection of small surfaces [33], and in some cases where water and soap are not available, the recommended alcohol is ethanol with a concentration of more than $70 \%$. Frequent use of alcohol removes the lipid from the skin and causes dryness [33]. In the most recent version, the benzalkonium chloride and chlorhexidine are still ineffective on coronaviruses unless $70 \%$ ethanol is added to these compounds. Chloroxylenol is in the list of ineffective compounds on coronaviruses [36]. It is very dangerous to use methanol, which is known as industrial alcohol. Methanol or industrial alcohol may be absorbed through the skin and respiratory tract, which can damage the respiratory system, kidneys, and can cause blindness. It should be noted that the CDC recommended non-smoking and non-use of alcohol because smoking increases the concentration of nicotine in the blood, which can lead to vasospasm and premature hypoxia in the organs. In particular, a decrease in oxygen in the respiratory tract and visceral damage can impair immunity. Drinking too much alcohol can damage the digestive system, liver, and brain cells and weaken the body's immune system.

\subsection{Disinfection of food and sterilization of bread}

Food and its use, depending on the type of cooking and use, can cause concerns about the transmission of COVID-19. Although the virus infects the lungs and is mostly transmitted through the respiratory transmission and through contact with released aerosols from the patient, the final respiratory manifestations are dangerous, and its transmission through meat and raw foods should be considered as an important precaution since animals have been first identified as the main origin of COVID-19. For example, meat should be completely cooked. According to the WHO Safe Shopping Protocol, after purchase, the materials must be thoroughly washed if possible [37]. Other foods, such as nuts and foods that cannot be cooked, should be (re)heated to destroy the virus before use [38].

Bread is one of the main components of the family food basket. Considering that there is a possibility of contamination of bread through contaminated hands, it is recommended to heat the bread before consumption. Because research has shown that COVID-19 is sensitive to heat and stays at a room temperature for up to 9 days, but is less resistant at temperatures above $30^{\circ} \mathrm{C}[8]$. Freezing bread has no effect on eliminating the virus, as it can survive for several years at temperatures up to $-60^{\circ} \mathrm{C}$, and, in fact, cold strengthens the virus. The results of a recent study published by The Lancet showed that the virus is highly resistant at $-4^{\circ} \mathrm{C}$, and the lowest decrease in the number of viruses was observed after 14 days [39]. Virus molecules are very stable in the cold and in air conditioning systems in homes and cars. They also need darkness and moisture to be more stable. Therefore, low-water, dry, hot, and bright environments lead to a faster destruction [26].

\subsection{Quarantine}

Quarantine is known to be an important step in the onset of a widespread epidemic and is the most effective way to stop the chain of an unknown contagious 
disease for which a vaccine and safe treatment has not yet been developed [19]. The duration of quarantine varies from source to source, but in most cases two weeks of quarantine are recommended (depending on the incubation period) [40]. However, there is a possibility of longer incubation periods for some people, and even after mild involvement it is possible to transmit the disease; thus, some countries have instated longer quaraintine periods. Based on the results of an article published in The Lancet Global Health, a mathematical model examines the control of COVID-19 infection by isolating individuals and examining the history of contact with infected individuals. It was stated that in most scenarios isolating individuals and reviewing and following up the contact history with infected individuals is sufficient to control the virus over a three month period. If there is a long delay between the onset of symptoms and isolation, the chances for disease control are decreased [41]. The results of other studies have shown that the centralized quarantine of patients in temporary hospitals or hotels and dormitories effectively stop the epidemic. Home quarantine, staying at home, and reducing public transportation also help but are not effective enough because they transmit the virus to patients at home, and the cycle of prevalence in society continues [42]. Ending a quarantine is possible depending on the circumstances. The final CDC rule for ending home quarantine is as follows for three different groups [22].

The first group: test-based group (the PCR-positive group). If they do not have a fever without taking any antipyretic and after relieving respiratory symptoms (i.e. cough, shortness of breath, etc.) and after two negative PCR tests were taken with a time interval of more than 24 hours, the quarantine does not have to be continued.

The second group: non-test-based group (a group that does not have an initial PCR test or calculates onset of the disease based on time). If they do not have a fever without taking any antipyretic for 72 hours and at least three days after the removal of respiratory symptoms (i.e. cough, shortness of breath, etc.) and seven days after the onset of symptoms, the quarantine does not have to be continued.

The third group: the group whose PCR test is positive but has no symptoms. If seven days have passed since the positive test and no symptoms were observed, the quarantine does not have to be continued.

The results of scientists in Hong Kong have shown that even people who recovered can transmit
COVID-19 through nasal secretions and saliva for up to 25 days, so even after recovery social distance must be observed [22].

\subsection{Observance of social distancing}

Maintaining social distance means staying at home, and not observing a distance of 1.5 meters. According to results published by the University of Sydney, Australia, if $90 \%$ of people observe social distancing, COVID-19 will be controlled within three months, and if only $70 \%$ of people observe social distaning, control will not be possible [43]. As recommended by the CDC, individuals should stay away from sick people, avoid direct contact with people in the community as much as possible, and use a mask [33]. According to Chinese researchers, COVID-19 can travel more than 4.5 meters and stays in the air for $30 \mathrm{~min}$ [44]. The results published by the United States have shown that social distancing has been largely effective in controlling the prevalence of COVID-19, and individuals should stay at home if they have symptoms and visit a health center if needed [33]. It should be noted that COVID-19 may be present in various societies and countries for a long time, and it is not possible to prolong quarantine, so smart social distancing should be observed.

\subsection{Return to work for people with COVID-19}

Return to work for the people with COVID-19 is possible when there is no possibility of transmission of the virus. In other words, when the person is not a carrier of the disease, the other employees are safe. According to the CDC's health protocols, people with COVID-19, after treatment and physical and paraclinical examinations by a physician, can return to work according to the recommended and standard algorithm. According to the form prepared in the study by a group of experts from the Health and Workplace Center of the Ministry of Health of Iran, the information of the individual will be recorded, and feedback will be provided to the employer. Examinations include examining the respiratory, cardiovascular, renal, and hepatic systems of the individual $[45,46]$.

\subsection{Observing the principle of prevention in clinical settings}

An important part of observing the principles of prevention is related to health care staff and all 
areas of work that are directly related to COVID-19 patients and includes proper use of personal protective equipment, observance of disinfection and sterilization principles during the transfer of patients, treatment process, performing diagnostic tests, laboratories, clothing and equipment used in the treatment process of patients, and the process of disposing of waste contaminated with COVID-19. Some treatments, such as tracheal intubation, non-invasive ventilation, cardiopulmonary resuscitation, manual ventilation before intubation, and bronchoscopy, have been associated with an increased risk of COVID-19 transmission due to the possibility of aerosol production. It should be ensured that health care workers who perform these procedures take precautions [2].

Therefore, the N95 mask recommended by NIOSH (EU FFP2 or its equivalent) should be used. When using disposable respiratory masks, staff should always do a seal and leak test. It should be noted that facial hair can prevent proper breathing. Eye protection (i.e. glasses, face shield), long-sleeved clothing, and clean gloves should be used. If the gown is not resistant to liquids, a waterproof apron should be used [2, 47]. It is worth noting that the health care staff is not limited to nurses and physicians, and includes all individuals, such as physicians, nurses, paramedics, emergency medical staff, diagnostic laboratory staff, and service personnel. According to the New York Times, the highest risk of infection is related to general physicians, laboratories, pharmacies, and radiology, which have the closest contact with COVID-19 patients and are placed in the next rank. It is also recommended that health care staff should take off their protective clothing immediately after finishing work, and take off their gloves and immediately wash their hands with water and soap or disinfect them by alcohol-based disinfectants. Moreover, individuals should not touch the mask with contaminated hands. In all work environments, in addition to training employees, sufficient detergents and disinfectants must be provided. All high-touch surfaces and objects should be sterilized and disinfected on a regular basis [33]. The results of the study showed that the most sensitive people to COVID19 are family members and relatives of the patient, elderly people, and, of course, medical staff. These three groups need quarantine, careful care, and protective equipment. Medical personnel is among the most susceptible carriers of the disease in the community, and if they are not well protected, they can transmit the virus first to their families and then to the whole community. Investing in these people's protective equipment and separating them from public communications is one of the priorities in combating the spread of the disease [48].

Increasing referrals to medical centers leads to an increase in the workload of health and medical staff. One of the consequences of this increase is fatigue and burnout, which leads to a decrese in the efficiency of employees and quality of services. On the other hand, high workload and a lack of sleep reduce the physical decline of healthcare professionals, and due to the high infection in the hospital, the risk of getting infected by COVID-19 is increased in the staff. Increasing the risk of infection causes the loss of community professionals. So far, statistics show that many doctors, nurses and service personnel have died in countries all over the world [49]. Also, the increase in the number of patients due to limited medical equipment led to difficulty in providing services, such as the lack of ventilators in Italy that led to the triage of patients [50].

\subsection{Principles of prevention in the patient transfer or transfer and burial of corpses with COVID-19}

In addition to observing the prevention rules in the transfer or treatment of suspected or ill people, it is important to observe the preventive rules in the transfer of sick people and the and corpses of COVID19 patients. Emergency medical personnel must use personal protective equipment when transporting a patient. If the sent unit announces that the patient is suspected of having COVID-19, the team sent must use the appropriate PPE before entering the site. If there is no information that the patient is suspected of having COVID-19, the sent team should be cautious when examining the patient with symptoms of a respiratory infection and, if possible, an initial evaluation should be initiated at a distance of 1 meter from the patient. Contact with the patient should be kept to a minimum until (s)he wears a surgical mask. If the patient is not suspected of having COVID-19, medical emergency personnel should follow standard procedures and use appropriate PPE to assess the patient with the respiratory infection. For transportation, the number of personnel, who provides essential services, should be limited so that the least number of people are at risk. Ambulance should only comprise of an isolated driver, and patient compartments, doors and windows should be closed. If an ambulance without an isolated driver compartment and ventilation is used, its window in the driver's area should be 
open, and the rear exhaust fan should be used at the highest setting; this will lead to a negative pressure gradient in the patient's area [51]. Preparation, washing, funeral, and burial of corpses require compliance with health requirements. The funeral and burial of COVID-19 patients' corpses should be carried out based on health principles so that people who provide transportation and burial do not get infected. It is also recommended to use waterproof PPE when washing the corpse and protective clothing with long sleeves should be worn during transport and burial. Even in cases where the autopsy of the deceased is required, all hygiene principles should be observed to prevent the spread of COVID-19 through the aerosols and droplets from the autopsy [52].

\subsection{The need for a face mask}

Studies have shown that the use of masks has a positive effect on the prevention of COVID-19 [53]. However, issues such as the frequency of mask use and the type of appropriate mask are a concern. It should be noted that surgical masks are made for single use. Also, respiratory masks are usually discarded after use, but they can be reused for a limited time unless there there is a risk of infection due to the deposition of infectious particles on the surface (for example, when the mask is used to care for patients with tuberculosis, it is acceptable to use respiratory masks several times if it is worn by the same person).

When respiratory masks are soaked with body fluids and can no longer be properly fitted to the face, or when breathing becomes difficult through the respiratory mask, they should be discarded. It should be noted that due to the high infection of the masks, it is necessary to discard them after use during a treatment procedure. However, an alternative approach, such as long-term use of the mask, has been recommended. By employing a surgical mask or wearing a clean face shield, infection can be prevented. In cases of severe shortages of surgical and respiratory masks, as was the case during the COVID-19 epidemic, a number of methods can be considered to sterilize the used masks. However, it should be noted that the mask is usually not used several times, and its disinfection with alcohol or washing is not very efficient, and even when washed, the efficiency is greatly reduced.

Studies have shown that cloth masks are less protective than surgical masks and may even increase the risk of infection due to moisture, fluid release, and virus retention. Overall, conventional fabric masks do not provide protection against respiratory viruses and are not recommended for use $[29,54]$. In the cases of a severe shortage of personal protective equipment and only in the absence of access to surgical or respiratory masks, homemade fabric masks (for example, scarves or T-shirts) are recommended by the US CDC as the last temporary solution until the standard PPE is achieved $[29,30]$.

In China, people living in Wuhan were obliged to wear a mask. However, some WHO recommendations have emphasized that the use of a mask is only necessary for people with symptoms and people working in health centers [55]. Due to the fact that some carriers are asymptomatic, $\mathrm{CDC}$ in its latest guidelines recommended that everyone should cover their respiratory area to avoid the spread of the infection due to respiratory droplets in places where it is not possible to observe the social distance, such as shops, pharmacies, etc. According to this guide, any simple fabric coverage that can prevent the release of respiratory droplets from carriers and asymptomatic people is recommended. It should be noted that these masks do not substitute surgical masks and N95 are only recommended for public environments [30].

\subsection{Prevention by controlling the spread of COVID-19 infection in the environment}

According to an article in the journal Science, washing and spraying streets and public places with disinfectants have no effect on preventing the spread of COVID-19 because the initial disinfection conditions, including adequate contact time, proper disinfection and repetition cannot be provided correctly and may lead to respiratory complications for people as well as environmental effects [56]. Therefore, targeted disinfection is recommended with a focus on high-touch and specific surfaces such as public places, public transportation systems, waste collection tanks, waste transfer stations, and waste collection and transportation facilities.

Respiratory masks and infected gloves are disposable, and studies have indicated the infection of saliva and nasal of COVID-19 patients. Release of this infected protective equipment in the environment and contact with these infected materials or contact with infected clothing and wipes impregnated with these biological liquids is dangerous; thus, they should be disposed properly after use, and infectious hospital waste should also be disposed of in accordance with hazardous waste disposal protocols. On the other hand, the production of this volume of infec- 
tious waste, in addition to the problem of standard sanitary disposal, will become a major challenge that pollutes the environment. Another positive achievement of the volume of visits to medical centers will be the reduction of waste production and environmental protection. Based on this, it can be said that if home quarantine is voluntarily observed, in addition to reducing the risk of COVID-19, and reducing the number of visits to medical centers, it will be associated with another positive achievement, which is the reduction of waste production and the development of environmental protection.

Another environmental consequence of COVID19 is an increase in the burden of biological contamination on hospital wastewater of COVID-19 patients' rehabilitation centers and mortuaries. The persistence of the virus in sewages, which is a sign of an epidemic in an area, can be important and can lead to catastrophic infectious diseases during floods and other crises, and require special guidelines for disinfection and treatment of wastewater in the event of epidemic crises. All wastewaters released from bathrooms, sinks, handwashing points, and laundries should be properly treated before infiltration [2], and the stabilization pond is an effective method because the retention time of more than 20 days along with sunlight destroys pathogens. If no action is taken to eliminate the virus, a final disinfection step is performed; for this purpose, hygienic principles must be observed to prevent the infection of the treatment plant workers. Those workers should wear appropriate PPE (i.e. protective clothing, gloves, boots, goggles or protective shields, and masks), observe frequent hand hygiene, and avoid touching the eyes, nose, or mouth with unwashed hands [23].

\subsection{Ambiguity in immunity after infection}

Re-infection of sick people is one of the ambiguities and points of doubt of scientists and researchers. Reports indicate that some patients are re-infected by COVID-19 after recovery [57], and it was predicted that $14 \%$ of recovered people would be at risk for re-infection and there is the possiblity of a person's involvement with a new form of the diseae due to the highly mutable structure of the virus and the conditions of its changes. Moreover, the treatment method is the injection of plasma taken from the recovered people into sick people's bodies $[58,59]$. This method is very expensive but has had good results in China. In addition, COVID-19 treatment with stem cells has recently been proposed in Iran [60]. A study con- ducted on monkeys who were exposed to COVID-19 for the second time showed that they do not become infected again. But it is not yet clear how long this safety period will last [61]. The collective epidemic approach and the creation of immunity of the whole society after the infection is completely rejected and dangerous. Because, in addition to the unprecedented killing of people, it imposes a heavy burden of medical referrals on the treatment system. Furthermore, as mentioned, it cannot be said with certainty that those who have been infected will not get infected again. This has been evaluated in several studies, and re-infection of a number of recovered patients was reported.

\subsection{Applying preventive instructions and rules}

Passenger traffic in different cities also helps to spread the infection in all parts of a city or country and increases the risk of COVID-19 infection. With the full quarantine of Wuhan, China has taken every necessary step to prevent any unnecessary traffic inside and outside of the city and has prevented the spread of the disease to other parts of China. This model was calibrated based on international reports about COVID-19, and it shows that many infected travelers entered most Chinese cities before the prohibition of travel on January 23, 2020. The travel quarantine of Wuhan has delayed the overall progress of the epidemic in China by only 3 to 5 days, but it has had a more significant impact on an international scale as it led to the reduction of the incidence of COVID19 by almost $80 \%$ by mid-February. The results of the modeling also show that travel restrictions to and from China, consistently up to $90 \%$, slightly affect the epidemic path unless it can be accompanied by a $50 \%$ or more reduction in transmission in the community [62].

In China and many other countries, including the United States, travel screening laws have been enacted to control the spread of the disease [1]. Some strict rules, such as fines for unnecessary leaving home and attending public places, which is considered a violation of quarantine laws, were also imposed in many countries, including Italy, Spain, and the United Kingdom. Moreover, in some countries, not reporting a sick person is subject to penalty. In China, a multi-dollar prize was awarded to people who reported people with COVID-19. Some governmental measures, such as screening and detection of the disease, in addition to assessing community conditions and quickly identifying people in need of 
medical care, will help to reduce referrals and the infection load caused by the presence in hospital settings. However, due to the presence of asymptomatic carriers that may not be detected even in the screening program, observing social distance is the best and most effective way to prevent an infection. In South Korea and Germany, it was possible to test a large number of people, consequently, to provide timely treatment and to reduce the risk of human exposure by isolating patients from healthy individuals. Statistics show that, in spite of a large number of identified patients. In Germany, mortality rates have been very low because of timely identification and action. Moreover, they could conduct 200,000 to 1 million COVID-19 tests a day. Dr. Dalglish, Professor at the Johns Hopkins School of Health, described how countries in the Far East, such as China, Korea, Singapore, and Taiwan, have responded effectively, quickly, and even creatively to these challenges, based on to their experiences with SARS and animal-borne flu. According to him, even the performance of weaker countries (such as African countries) has been remarkable so far. While COVID19 deceived developed countries such as the United States and the United Kingdom, and challenged the reputation of experience and expertise in these countries so that they are the first and second ranks in terms of the number of infected cases in the world, respectively [63]. China's successful experiments in disease control have been the result of several effective and timely measures that have generally included early diagnosis, early reporting, early isolation, and early treatment and have prevented the spread of the disease throughout China. Some of these measures have resulted from an effective integrated command system and the application of strict restrictions [61].

Accordingly, the role of the government, in addition to timely and accurate training and information is very important in formulating deterrent laws and appropriate measures based on the opinion of experts and can be very effective in controlling the spread and stopping the chain [64]. The laws related to prohibiting the activities of various guilds, except for jobs and guilds producing essential necessities such as small grocery stores and pharmacies, is one the law that is worth mentioning. Of course, it should be noted that the reopening of all guilds and the return to the activities of the guilds should be based on scientific and technical evaluation of the existing conditions and should be in accordance with the principles of health and prevention until the final eradication of COVID-19.

\subsection{Challenges of the need for public information and public education}

Timely training and information will increase public awareness and lead to informed decisions by the public to reduce the prevalence and to prevent casualties and costs caused by the multiplicity of patients in treatment centers. One of China's most valuable measures has been the timely information and training and use of cyberspace capacity to reduce stress caused by the COVID-19 crisis [65]. Because without the cooperation of the public, overcoming the conditions and controlling the crisis will not be possible [57]. The growing and uncontrollable outbreak in Italy has been due to ignoring the issue and perhaps the unpreparedness and unpredictability of the COVID-19 outbreak in the country, which has led to a high number of casualties [66]. It is worth noting that due to the unknown dimensions of the virus and the change and updating of information on how to care and prevent during research, and the guidelines of the WHO and CDC, a lot of effort is needed for training different people, ranging from children to elderly and from people with disabilities to ordinary people. In the context of educational challenges, it is important not to underestimate and neglect the virus. If China, which first discovered and detected the disease and became involved in its epidemic, provided more accurate information, as well as the importance and nature of the virus to the entire world, perhaps the international community could be able to control the origin of this virus and the entire world would not be stuck in a pandemic. Of course, in the field of educational challenges, the issue of transferring false and incorrect information from social networks and non-scientific sources, in addition to the unknown aspects of the virus and updating the information of competent institutions, has lead to misleading or misbehaving. An example is alcohol consumption and the number of deaths caused by its consumption in Iran. In this context, people's confusion about how to get the right information can also lead to stress in the society and a sense of fear or distrust in the society.

\section{Limitations}

Many studies are still being performed in this field, and some cases are being updated. Therefore, the lack of valid scientific studies and evidence can challenge the scope of this research. Different genetic mutations of COVID-19 and the type of reaction of different 
people to the virus depend on individual differences, individual resistance, and underlying diseases and a dose of exposure to the virus and other factors are among the ambiguities and important challenges of this pandemic, which will certainly be identified by performing more detailed studies.

\section{Conclusion}

Since we are faced with a global COVID-19 outbreak, which is rapidly spreading, and there is no vaccine for prevention of the disease (at the time of writing of this article), there are still many ambiguities about the origin of the disease and the different ways of transmission. Issues about individual susceptibility to the virus and the severity of the disease in different individuals, as well as immunity after infection, are still unclear. Thus, the policy of quarantine for a few weeks and lockdown of tourism, cultural places, and parks are necessary, and observing personal hygiene is the most effective way to stop the transmission chain.

Therefore, observing personal hygiene and the correct principles of handwashing, disinfecting hightouch surfaces, observing social distance, and not attending public places are recommended as preventive measures. Some important measures related to applying the obstructive rules and regulations by decision-making bodies to strengthen telecommuting and to reduce the presence of people in the society and to prevent unnecessary activities have an imperative role in controlling the situation and reduce infection. We hope that after passing the peak period in each country and its control, the disease will later become endemic and sporadic in the entire world, and we will go through such crises.

\section{Conflict of interest}

None to report.

\section{References}

[1] Rothan HA, Byrareddy SN. The epidemiology and pathogenesis of coronavirus disease [COVID-19] outbreak. Journal of Autoimmunity. 2020:102433.

[2] Organization WH. Severe acute respiratory infections treatment centre: practical manual to set up and manage a SARI treatment centre and a SARI screening facility in health care facilities. World Health Organization, 2020.
[3] Munster VJ, Koopmans M, van Doremalen N, van Riel D, de Wit E. A novel coronavirus emerging in China-key questions for impact assessment. New England Journal of Medicine. 2020;382(8):692-4.

[4] Zhou P, Yang X-L, Wang X-G, Hu B, Zhang L, Zhang W, et al. Discovery of a novel coronavirus associated with the recent pneumonia outbreak in humans and its potential bat origin. BioRxiv. 2020.

[5] Guo Y-R, Cao Q-D, Hong Z-S, Tan Y-Y, Chen S-D, Jin $\mathrm{H}-\mathrm{J}$, et al. The origin, transmission and clinical therapies on coronavirus disease 2019 [COVID-19] outbreak-an update on the status. Military Medical Research. 2020;7(1): $1-10$.

[6] Menachery VD, Yount Jr BL, Debbink K, Agnihothram S, Gralinski LE, Plante JA, et al. A SARS-like cluster of circulating bat coronaviruses shows potential for human emergence. Nature Medicine. 2015;21(12):1508.

[7] Andersen KG, Rambaut A, Lipkin WI, Holmes EC, Garry RF. The proximal origin of SARS-CoV-2. Nature Medicine. 2020.

[8] Kampf G, Todt D, Pfaender S, Steinmann E. Persistence of coronaviruses on inanimate surfaces and its inactivation with biocidal agents. Journal of Hospital Infection. 2020.

[9] Huang C, Wang Y, Li X, Ren L, Zhao J, Hu Y, et al. Clinical features of patients infected with 2019 novel coronavirus in Wuhan, China. The Lancet. 2020;395(10223):497-506.

[10] Bi Q, Wu Y, Mei S, Ye C, Zou X, Zhang Z, et al. Epidemiology and Transmission of COVID-19 in Shenzhen China: Analysis of 391 cases and 1,286 of their close contacts. MedRxiv. 2020.

[11] Available from: https://www.who.int/emergencies/diseases/ novel-coronavirus-2019 [press release]. 2020.

[12] https://www.euronews.com/2020/03/27/coronavirus-infrance-healthy-16-year-old-dies-of-covid-19 Ai.

[13] https://www.euronews.com/2020/03/31/coronavirusdoctors-devastated-as-covid-19-claims-life-of-12-yearold-girl-in-belgium Ai.

[14] Chen H. Susceptibility of ferrets, cats, dogs, and different domestic animals to SARS-coronavirus-2. bioRxiv. 2020

[15] National Geographic. Available from: https://www.national geographic.com/animals/2020/04/tiger-coronaviruscovid19-positive-test-bronx-zoo/?utm_source=Nature+ Briefing\&utm_campaign $=\mathrm{d} 04639 \mathrm{c} 3 \mathrm{df}-$ briefing-dy-20200 406\&utm_medium=email\&utm_term=0_c9dfd39373d04639c3df-43592209

[16] Betsch C, Wieler L, Bosnjak M, Ramharter M, Stollorz V, Omer S, et al. Germany COVID-19 Snapshot MOnitoring [COSMO Germany]: Monitoring knowledge, risk perceptions, preventive behaviours, and public trust in the current coronavirus outbreak in Germany. 2020.

[17] Lai C-C, Shih T-P, Ko W-C, Tang H-J, Hsueh P-R. Severe acute respiratory syndrome coronavirus 2 [SARS-CoV-2] and corona virus disease-2019 [COVID-19]: the epidemic and the challenges. International journal of antimicrobial agents. 2020:105924.

[18] doshmangir MA, Gholipour KA, S A. Strategies of East Asian Countries in the Effective Confrontation with Quaid19: Lessons Learned for Iran. Journal of Management Strategies in the Health System. 2020;4(4):370-3.

[19] Khan N, Fahad S, Faisal S, Naushad M. Quarantine Role in the Control of Corona Virus in the World and Its Impact on the World Economy. Available at SSRN 3556940. 2020.

[20] Organization WH. Infection prevention and control during health care when COVID-19 is suspected: interim guidance, 19 March 2020. World Health Organization, 2020. 
[21] Lauer SA, Grantz KH, Bi Q, Jones FK, Zheng Q, Meredith HR, et al. The incubation period of coronavirus disease 2019 [COVID-19] from publicly reported confirmed cases: estimation and application. Annals of internal medicine. 2020.

[22] To KK-W, Tsang OT-Y, Leung W-S, Tam AR, Wu T-C, Lung DC, et al. Temporal profiles of viral load in posterior oropharyngeal saliva samples and serum antibody responses during infection by SARS-CoV-2: an observational cohort study. The Lancet Infectious Diseases. 2020.

[23] Organization WH. Water, sanitation, hygiene and waste management for COVID-19: technical brief, 03 March 2020. World Health Organization, 2020.

[24] CNBC. [https://www.cnbc.com/2020/03/16/who-consid ers-airborne-precautions-for-medical-staff-after-studyshows-coronavirus-can-survive-in-air.html] 2020.

[25] van Doremalen N, Bushmaker T, Morris DH, Holbrook MG, Gamble A, Williamson BN, et al. Aerosol and Surface Stability of SARS-CoV-2 as Compared with SARS-CoV-1. New England Journal of Medicine. 2020.

[26] Gallagher T, Escarmis C, Buchmeier M. Alteration of the $\mathrm{pH}$ dependence of coronavirus-induced cell fusion: effect of mutations in the spike glycoprotein. Journal of virology. 1991;65(4):1916-28.

[27] Ong SWX, Tan YK, Chia PY, Lee TH, Ng OT, Wong MSY, et al. Air, surface environmental, and personal protective equipment contamination by severe acute respiratory syndrome coronavirus 2 [SARS-CoV-2] from a symptomatic patient. Jama. 2020.

[28] Nagai A, Hirata M, Kamatani Y, Muto K, Matsuda K, Kiyohara Y, et al. Overview of the BioBank Japan Project: study design and profile. Journal of epidemiology. 2017;27[Supplement_III]:S2-S8.

[29] MacIntyre CR, Seale H, Dung TC, Hien NT, Nga PT, Chughtai $\mathrm{AA}$, et al. A cluster randomised trial of cloth masks compared with medical masks in healthcare workers. BMJ open. 2015;5(4):e006577.

[30] Adams J. Recommendation regarding the use of cloth face coverings, especially in areas of significant communitybased transmission. 2020.

[31] Cascella M, Rajnik M, Cuomo A, Dulebohn SC, Di Napoli R. Features, evaluation and treatment coronavirus [COVID19]. StatPearls [Internet]: StatPearls Publishing; 2020.

[32] Bai Y, Yao L, Wei T, Tian F, Jin D-Y, Chen L, et al. Presumed Asymptomatic Carrier Transmission of COVID-19. JAMA. 2020.

[33] all WZae. The Coronavirus Prevention Handbook: 101 Science-Based Tips That Could Save Your Life Kindle Edition. China: Skyhorse [March 10, 2020]; 2020.

[34] Kenters N, Eikelenboom-Boskamp A, Hines J, McGeer A, Huijskens E, Voss A. Product dose considerations for realworld hand sanitiser efficacy. American Journal of Infection Control. 2020.

[35] Wu Y, Guo C, Tang L, Hong Z, Zhou J, Dong X, et al. Prolonged presence of SARS-CoV-2 viral RNA in faecal samples. The Lancet Gastroenterology \& Hepatology. 2020;5(5):434-5.

[36] McIntosh K, Hirsch MS, Bloom A. Coronavirus disease 2019 (COVID-19): Epidemiology, virology, and prevention. Available from: uptodate.com/contents/coronavirus-disea se-2019-covid-19-epidemiology-virology-and-prevention, Accessed Feb 18, 2020

[37] Seymour N, Yavelak M, Christian C, Chapman B, Danyluk M. COVID-19 FAQ for Food Service: Receiving and Food Packaging. EDIS. 2020;2020[2].
[38] Baluja A, Arines J, Vilanova R, Bao-Varela C, Flores-Arias MT. UV light dosage distribution over irregular respirator surfaces. Methods and implications for safety. medRxiv. 2020.

[39] Chin A, Chu J, Perera M, Hui K, Yen H-L, Chan M, et al. Stability of SARS-CoV-2 in different environmental conditions. medRxiv. 2020.

[40] McIntosh K. Coronavirus disease 2019 [COVID-19]. UpToDate. 2020.

[41] Hellewell J, Abbott S, Gimma A, Bosse NI, Jarvis CI, Russell TW, et al. Feasibility of controlling COVID-19 outbreaks by isolation of cases and contacts. The Lancet Global Health. 2020;8(4):e488-e96.

[42] Hellewell J, Abbott S, Gimma A, Bosse NI, Jarvis CI, Russell TW, et al. Feasibility of controlling 2019-nCoV outbreaks by isolation of cases and contacts. medRxiv. 2020:2020.02.08.20021162.

[43] Chang SL, Harding N, Zachreson C, Cliff OM, Prokopenko M. Modelling transmission and control of the COVID-19 pandemic in Australia. arXiv preprint arXiv:200310218. 2020.

[44] Han Y, Lam JC, Li VO, Guo P, Zhang Q, Wang A, et al. The Effects of Outdoor Air Pollution Concentrations and Lockdowns on Covid-19 Infections in Wuhan and Other Provincial Capitals in China. 2020.

[45] Control CfD, Prevention. Interim US Guidance for Risk Assessment and Public Health Management of Healthcare Personnel with Potential Exposure in a Healthcare Setting to Patients with Coronavirus Disease [COVID-19]. March 7, 2020. 2020.

[46] Guidance CI. Interim Guidance for Businesses and Employers to Plan and Respond to Coronavirus Disease 2019 [COVID-19], February 2020. 2020.

[47] Organization WH. Perform a particulate respirator seal check. Geneva: World Health Organization; 2007. Avalable in: https://www.who.int/csr/resources/publications/ respiratorsealcheck/en/. 2007.

[48] Li W, Zhang J, Xiao S, Sun L. Characteristics of health worker fatality in China during the outbreak of COVID-19 infection. Journal of Infection. 2020.

[49] Lancet T. COVID-19: protecting health-care workers. Lancet [London, England]. 2020;395(10228):922.

[50] Truog RD, Mitchell C, Daley GQ. The Toughest TriageAllocating Ventilators in a Pandemic. New England Journal of Medicine. 2020

[51] CDC. Available from: https://www.cdc.gov/coronavirus/ 2019-ncov/hcp/index.html. 2020.

[52] Organization WH. Infection prevention and control of epidemic-and pandemic-prone acute respiratory infectionsin health care. Available from: https://www.who.int/csr/ bioriskreduction/infection_control/publication/en/. 2020.

[53] Leung NH, Chu DK, Shiu EY, Chan K-H, McDevitt JJ, Hau BJ, et al. Respiratory Virus Shedding in Exhaled Breath and Efficacy of Face Masks.

[54] Romagna E, Giulia FV, Adige TA, d'Aosta V, Nazionale C, Romagna ABCCE, et al. Covid-19. E'possibile sterilizzare le maschere FFP2/FFP3?

[55] Organization WH. Advice on the use of masks in the community, during home care and in healthcare settings in the context of the novel coronavirus [2019-nCoV] outbreak: interim guidance, 29 January 2020. World Health Organization, 2020.

[56] Hashemi H, Amin MM, Ebrahimi A, Rezaie R, Safari M. Evaluation of health, environmental, economic and technical aspects of disinfection of WWTP effluent in the north of 
Isfahan with UV instead of chlorine. Scientific Journal of Kurdistan University of Medical Sciences. 2012;16(4):50-9.

[57] Lan L, Xu D, Ye G, Xia C, Wang S, Li Y, et al. Positive RT-PCR test results in patients recovered from COVID-19. Jama. 2020.

[58] Duan K, Liu B, Li C, Zhang H, Yu T, Qu J, et al. Effectiveness of convalescent plasma therapy in severe COVID-19 patients. Proceedings of the National Academy of Sciences. 2020.

[59] Cunningham AC, Goh HP, Koh D. Treatment of COVID-19: old tricks for new challenges. Springer; 2020.

[60] Orleans L, is Vice H, Manchikanti L. Expanded Umbilical Cord Mesenchymal Stem Cells [UC-MSCs] as a Therapeutic Strategy In Managing Critically Ill COVID-19 Patients: The Case for Compassionate Use. Pain Physician. 2020;23:E71-E83.

[61] Kupferschmidt K, Cohen J. China's aggressive measures have slowed the coronavirus. They may not work in other countries. Science. 2020;2.
[62] Chinazzi M, Davis JT, Ajelli M, Gioannini C, Litvinova $\mathrm{M}$, Merler S, et al. The effect of travel restrictions on the spread of the 2019 novel coronavirus [COVID-19] outbreak. Science. 2020:eaba9757.

[63] Dalglish SL. COVID-19 gives the lie to global health expertise. The Lancet. 2020.

[64] Gros C, Valenti R, Valenti K, Gros D. Strategies for controlling the medical and socio-economic costs of the Corona pandemic. arXiv preprint arXiv:200400493. 2020.

[65] Zhang J, Wu W, Zhao X, Zhang W. Recommended psychological crisis intervention response to the 2019 novel coronavirus pneumonia outbreak in China: a model of West China Hospital. Precision Clinical Medicine. 2020;3(1):3-8.

[66] Onder G, Rezza G, Brusaferro S. Case-fatality rate and characteristics of patients dying in relation to COVID-19 in Italy. Jama. 2020. 